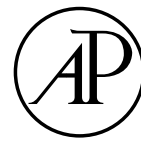

ACADEMIC PRESS
Available online at www.sciencedirect.com

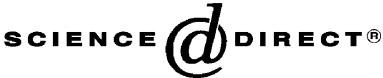

Journal of Computational Physics 186 (2003) 24-46
JOURNAL OF COMPUTATIONAL PHYSICS

www.elsevier.com/locate/jcp

\title{
High-order non-reflecting boundary scheme for time-dependent waves
}

\author{
Dan Givoli *,1, Beny Neta ${ }^{2}$ \\ Department of Mathematics, Naval Postgraduate School, 1141 Cunningham Road, Monterey, CA 93943, USA
}

Received 30 April 2002; received in revised form 3 December 2002; accepted 13 December 2002

\begin{abstract}
A new non-reflecting boundary scheme is proposed for time-dependent wave problems in unbounded domains. The linear time-dependent wave equation, with or without a dispersive term, is considered in a semi-infinite wave guide. The infinite domain is truncated via an artificial boundary $\mathscr{B}$, and a high-order non-reflecting boundary condition (NRBC) is imposed on $\mathscr{B}$. Then the problem is solved numerically in the finite domain bounded by $\mathscr{B}$. The new boundary scheme is based on a reformulation of the sequence of NRBCs proposed by Higdon. In contrast to the original formulation of the Higdon conditions, the scheme constructed here does not involve any high derivatives beyond second order. This is made possible by introducing special auxiliary variables on $\mathscr{B}$. As a result, the new NRBCs can easily be used up to any desired order. They can be incorporated in a finite element or a finite difference scheme; in the present paper the latter is used. The parameters appearing in the NRBC are chosen automatically via a special procedure. Numerical examples concerning a semi-infinite wave guide are used to demonstrate the performance of the new method.

Published by Elsevier Science B.V.
\end{abstract}

Keywords: Waves; High-order; Artificial boundary; Non-reflecting boundary condition; Higdon; Auxiliary variables; Finite difference

\section{Introduction}

Methods for the numerical solution of wave problems in unbounded domains have been developed since the 1970s [1]. They have been considered in various fields of application involving wave propagation, such as acoustics, electromagnetics, meteorology and solid geophysics. The main four types of methods that have emerged are: boundary integral methods, infinite element methods, absorbing layer methods and nonreflecting boundary condition (NRBC) methods. The present paper concentrates on the latter.

\footnotetext{
${ }^{*}$ Corresponding author. Tel.: 1-831-656-2758; fax: 1-831-656-2355.

E-mail addresses: givolid@aerodyne.technion.ac.il, dgivoli@nps.navy.mil (D. Givoli), bneta@nps.navy.mil (B. Neta).

${ }^{1}$ On leave (till August 2002) from Department of Aerospace Engineering and Asher Center for Space Research, Technion, Israel Institute of Technology, Haifa 32000, Israel. Tel.: +972-4-829-3818; fax: +972-4-823-1848.

${ }^{2}$ Tel.: 1-831-656-2758; fax: +1-831-656-2355.
} 
In the method of NRBCs, the infinite domain is truncated via an artificial boundary $\mathscr{B}$, thus dividing the original domain into a finite computational domain $\Omega$ and a residual infinite domain $D$. A special boundary condition is imposed on $\mathscr{B}$, in order to complete the statement of the problem in $\Omega$ (i.e., make the solution in $\Omega$ unique) and, most importantly, to ensure that no (or little) spurious wave reflection occurs from $\mathscr{B}$. This boundary condition is called a NRBC, although a few other names are often used too [2]. The problem is then solved numerically in $\Omega$. The setup is illustrated in Fig. 1. Fig. 1(a) pertains to an exterior problem outside of a scatterer or an obstacle. The artificial boundary $\mathscr{B}$ has a rectangular shape in the figure, although sometimes a smooth shape (like a circle in two dimensions or a sphere in three dimensions) is preferred. Fig. 1(b) describes a semi-infinite wave-guide problem. In the example shown, $\mathscr{B}=\Gamma_{\mathrm{E}}$ is a crosssection of the wave-guide which constitutes the east side of $\Omega$.

Naturally, the quality of the numerical solution strongly depends on the properties of the NRBC employed. In the last 25 years or so, much research has been done to develop NRBCs that after discretization lead to a scheme which is stable, accurate, efficient and easy to implement. See [3-5] for recent reviews on the subject. Of course, it is difficult to find a single NRBC which is ideal in all respects and all cases; this is why the quest for better NRBCs and their associated discretization schemes continues.

The late 1970s and early 1980s produced some low-order local NRBCs that become well-known, e.g., the Engquist-Majda NRBCs [6] and the Bayliss-Turkel NRBCs [7]. The late 1980s and early and mid 1990s have been characterized by the emerging of exact non-local NRBCs like those based on the Dirichlet-toNeumann (DtN) map [8,9] and on the difference potential method (DPM) [10,11], and by the invention of the perfectly matched layer (PML) [12]. More recently, high-order local NRBCs have been introduced. Sequences of increasing-order NRBCs have been available before (e.g., the Bayliss-Turkel conditions [7] constitute such a sequence), but they had been regarded as impractical beyond 2 nd or third order from the implementation point of view. Only since the mid 1990s, practical high-order NRBCs have been devised.

The first such high-order NRBC has apparently been proposed by Collino [13], for two-dimensional time-dependent waves in rectangular domains. Its onstruction requires the solution of the one-dimensional wave equation on $\mathscr{B}$. Grote and Keller [14,15] developed a high-order converging NRBC for the threedimensional time-dependent wave equation, based on spherical harmonic transformations. They extended this NRBC for the case of elastic waves in [16]. Sofronov [17] has independently published a similar scheme in the Russian literature. Hagstrom and Hariharan [18,19] constructed high-order NRBCs for the two- and three-dimensional time-dependent wave equations based on the analytic series representation for the outgoing solutions of these equations. It looks simpler than the previous two NRBCs. For time-dependent waves in a two-dimensional wave guide, Guddati and Tassoulas [20] devised a high-order NRBC by using rational approximations and recursive continued fractions. Givoli [21] has shown how to derive high-order NRBCs for a general class of wave problems, leading to a symmetric finite element formulation, In [22], this methodology was applied to the particular case of time-harmonic waves, using optimally localized DtN NRBCs.

In the context of artificial boundary treatment, wave problems can roughly be divided into four categories. These are, in order of difficulty:

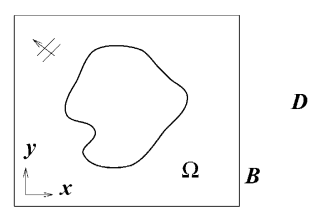

(a)

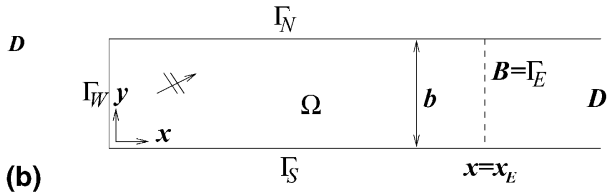

Fig. 1. Setup for the NRBC method: (a) an exterior scattering problem; (b) a semi-infinite wave-guide problem. 
(a) Linear time-harmonic wave problems.

(b) Linear time-dependent wave problems in non-dispersive homogeneous media.

(c) Linear time-dependent wave problems in dispersive and/or stratified media.

(d) Nonlinear time-dependent wave problems.

Linear time-harmonic waves have been treated extensively by NRBCs and absorbing layers, including exact NRBCs of the DtN type [4], various PML formulations (see, e.g. [23,24]), and converging high-order NRBCs (see, e.g. [22]). Time-dependent waves are considerably more difficult to handle from the artificialboundary perspective. However, some exact and high-order schemes have been devised in this case as well. These include the schemes proposed in [13-21] mentioned above, as well as a scheme based on the Kirchhoff formula for three-dimensional waves [25,26], an iterative converging local NRBC [27], semi-discrete DtN [28], time-dependent DtN [29], transform-based methods [30-32], and some variations of the above [33-35].

The presence of wave dispersion and/or medium stratification makes the time-dependent problem still more difficult as far as NRBC treatment is concerned. None of the high-order and exact NRBCs mentioned above has been designed to deal with these effects. Exact boundary conditions for the dispersive wave equation were developed by Hagstrom [5, p. 60], which was followed by limited experiments based on Padé approximations in [36]. Very recently, Navon et al. [37] developed a PML scheme for the dispersive shallow water equations. Nonlinear waves (with the nonlinearity extending to infinity) are, of course, the most difficult to handle. Some highly-accurate NRBCs have been proposed for specific classes of nonlinear wave problems (see references in the review papers [3,5,38]).

In the present paper we develop a high-order NRBC scheme for both dispersive and non-dispersive linear time-dependent waves. Wave dispersion appears in various applications. One important example is that of meteorological models which take into account the earth rotation [39]. Other examples include quantum-mechanics waves, the vibration of structures with rotational rigidity such as beams, plates and shells, the vibration of strings and membranes on an elastic foundation, acoustic wave propagation in a bubbly medium, and some nonlinear wave problems after linearization.

Higdon [40] proposed a sequence of NRBCs for the dispersive wave equation. In fact, these NRBCs were developed originally for non-dispersive waves [41-45], but Higdon [40] showed that they can be applied in the dispersive case too. The original implementation of the Higdon NRBCs is limited to low orders. Recently, we have proposed a new implementation method that allows the use of high-order discretized Higdon NRBCs [46]. However, this method differs from the original Higdon formulation only on the discrete level, not on the continuous level; thus, like the original Higdon scheme, it involves high normal and temporal derivatives, of increasing order. This has several disadvantages which we allude to in the next section. In addition, the computational effort required by the scheme devised in [46] grows exponentially with the order of the NRBC.

In the present paper, we reformulate the Higdon NRBCs on the continuous level in a completely new way. This formulation does not involve any high derivatives. This is made possible by introducing special auxiliary variables on $\mathscr{B}$. The new construction allows the easy use of a Higdon-type NRBC of any desired order, and can be incorporated in a finite element or a finite difference (FD) scheme. In the present paper we use FDs to discretize both the partial differential equation in $\Omega$ and the NRBC on $\mathscr{B}$. The computational effort required by the scheme grows only linearly with the order.

The approach used here is reminiscent of that of Hagstrom and Hariharan [18,19]. In both cases new high-order NRBCs are obtained from existing ones by eliminating all the high derivatives via the use of auxiliary variables. However, there are some significant differences between the two formulations:

- the Hagstrom-Hariharan conditions are constructed in cylindrical and spherical coordinates whereas the present conditions are constructed in Cartesian coordinates,

- the Hagstrom-Hariharan NRBCs are developed from the Bayliss-Turkel conditions, whereas here the starting point for the new NRBCs is the sequence of Higdon conditions. 
- The Hagstrom-Hariharan conditions are convergent in three dimensions and asymptotic in two dimensions whereas the present NRBCs are always convergent, albeit in the weaker sense discussed in Section 5.3 .

- The definition of the auxiliary variables in the Hagstrom-Hariharan formulation is rather complicated, whereas here they are defined via simple recursive relations.

- The NRBCs proposed here can be used equally well in the non-dispersive and dispersive cases, whereas the Hagstrom-Hariharan NRBCs were designed in [18,19] only for the non-dispersive case.

Although the proposed NRBCs are quite general, we restrict ourselves in this paper to the case of a semiinfinite wave guide (Fig. 1(b)), and do not treat the exterior scattering case (Fig. 1(a)) at all. The reason is that our formulation relies on Cartesian coordinates, and thus in the exterior case we have to deal with special conditions at the corners of $\mathscr{B}$ (see [47] for a discussion of this question for a certain family of NRBCs) and to verify that no instabilities develop there. While we firmly believe that the proposed scheme can be extended to this case, the investigation of the corner issue is not trivial and is left for future work.

Following is the outline of the rest of this paper. In Section 2 we state the problem under investigation. We also present the Higdon NRBCs and recall their properties. In Section 3 we reformulate the Higdon NRBCs, through the use of auxiliary variables, in a high-order way which does not involve high derivatives. The FD discretization scheme for this formulation is presented in Section 4. In Section 5 we discuss some computational issues, including the automatic choice of the NRBC parameters and the "exactness" of the NRBC scheme. In Section 7 we demonstrate the performance of the new method via some numerical examples concerning a semi-infinite wave guide. We conclude with some remarks in Section 7.

\section{Statement of the problem and the Higdon NRBCs}

We consider wave propagation in a two-dimensional channel or wave guide, as described in Fig. 1(b). A Cartesian coordinate system $(x, y)$ is introduced such that the wave-guide is parallel to the $x$ direction. The width of the wave-guide is denoted by $b$. In the wave guide we consider the linear inhomogeneous KleinGordon equation,

$$
\partial_{t}^{2} u-C_{0}^{2} \nabla^{2} u+f^{2} u=S .
$$

Here and elsewhere we use the following shorthand for partial derivatives:

$$
\partial_{a}^{i}=\frac{\partial^{i}}{\partial a^{i}} .
$$

In (1), $u$ is the unknown wave field, $C_{0}$ is the given reference wave speed, $f$ is the given dispersion parameter, and $S$ is the given wave source function. The $C_{0}$ and $f$ are allowed to be functions of location; however, it is assumed that outside a finite region (typically located near the west boundary $\Gamma_{\mathrm{W}}$ ) they do not depend on $x$ but only possibly on $y$. (Such $y$-dependence corresponds to a stratified medium.) The wave source $S$ is a function of location and time, but it is assumed to have a local support.

Eq. (1) describes, for example, the lateral vibration of a membrane strip on an elastic foundation, or the acoustic pressure in a dispersive medium (say, a linearized bubbly medium). Also, it can be shown that the linearized shallow water equations, with a flat bottom and zero initial conditions, reduce to (1), where $u$ is the water elevation above the reference level [39]. In the geophysical context, $f$ is called the Coriolis parameter and is related to the angular velocity of the earth.

On the south and north boundaries $\Gamma_{\mathrm{S}}$ and $\Gamma_{\mathrm{N}}$ we specify either the Dirichlet condition

$$
u=0 \text { on } \Gamma_{\mathrm{S}} \text { and } \Gamma_{\mathrm{N}},
$$


or the Neumann condition

$$
\partial_{y} u=0 \quad \text { on } \Gamma_{\mathrm{S}} \text { and } \Gamma_{\mathrm{N}} \text {. }
$$

In acoustics these correspond to "soft wall" and "hard wall" conditions, respectively. On the west boundary $\Gamma_{\mathrm{W}}$ we prescribe $u$ using a Dirichlet condition, i.e.,

$$
u(0, y, t)=u_{\mathrm{W}}(y, t) \text { on } \Gamma_{\mathrm{W}},
$$

where $u_{\mathrm{W}}(y, t)$ is a given function (incoming wave). At $x \rightarrow \infty$ the solution is known to be bounded and not to include any incoming waves. To complete the statement of the problem, the initial conditions

$$
u(x, y, 0)=u_{0}, \quad \partial_{t} u(x, y, 0)=v_{0},
$$

are given at time $t=0$ in the entire domain. We assume that the functions $u_{0}$ and $v_{0}$ have a local support.

We now truncate the semi-infinite domain by introducing an artificial east boundary $\mathscr{B} \equiv \Gamma_{\mathrm{E}}$, located at $x=x_{\mathrm{E}}$; see Fig. 1(b). This boundary divides the original semi-infinite domain into two subdomains: an exterior domain $D$, and a finite computational domain $\Omega$ which is bounded by $\Gamma_{\mathrm{W}}, \Gamma_{\mathrm{N}}, \Gamma_{\mathrm{S}}$ and $\Gamma_{\mathrm{E}} \mathrm{We}$ choose the location of $\Gamma_{\mathrm{E}}$ such that the entire support of $S, u_{0}$ and $v_{0}$ and the region of $x$-dependence of $C_{0}$ and $f$ are all contained inside $\Omega$. Thus, on $\Gamma_{\mathrm{E}}$ and in $D$, the homogeneous counterpart of (1) holds, i.e.,

$$
\partial_{t}^{2} u-C_{0}^{2} \nabla^{2} u+f^{2} u=0
$$

with $y$-dependent (or, as a special case, constant) coefficients $C_{0}^{2}$ and $f^{2}$, and the medium is initially at rest.

To obtain a well-posed problem in the finite domain $\Omega$ we need to impose a boundary condition on $\Gamma_{\mathrm{E}}$. This must be a NRBC so as to prevent spurious reflection of waves. We shall use a NRBC which is a reformulation of the Higdon NRBC [40]. The Higdon conditions were presented and analyzed in a sequence of papers [41-45] for non-dispersive acoustic and elastic waves, and were extended in [40] for the dispersive case. The Higdon NRBC of order $J$ is

$$
H_{J}:\left[\prod_{j=1}^{J}\left(\partial_{t}+C_{j} \partial_{x}\right)\right] u=0 \quad \text { on } \Gamma_{\mathrm{E}} .
$$

Here, the $C_{j}$ are parameters which have to be chosen and which signify phase speeds in the $x$-direction. The main advantages of the Higdon conditions are as follows:

- The Higdon NRBCs are very general, namely they apply to a variety of wave problems, in one, two and three dimensions and in various configurations. Moreover, they can be used, without any difficulty, for wave problems in dispersive and stratified media. Most other available NRBCs are either designed for non-dispersive homogeneous media (as in acoustics and electromagnetics) or are inherently of low order (as in meteorology and oceanography).

- The Higdon NRBCs constitute a sequence of conditions of increasing order. This, and the fact that no asymptotic approximation is involved in their construction, enables one in principle (leaving implementational issues aside for the moment) to obtain solutions with unlimited accuracy. See the discussion in Section 5.3.

- The boundary condition (8) is exact for all waves that propagate with an $x$-direction phase speed equal to either of $C_{1}, \ldots, C_{J}$. To see this, consider a wave which satisfies the wave equation (7). Such a wave has the form

$$
u=A Y_{n}(y) \cos k\left(x-C_{x} t+\psi\right),
$$

where

$$
C_{x}=\frac{\omega}{k},
$$


which is the $x$-direction phase velocity. In (9) and (10), $A$ is the wave amplitude, $\psi$ is its phase, $k$ is the $x$ component wave number, and $\omega$ is the wave frequency. The function $Y_{n}(y)$ in (9) is determined from the dependency of $C_{0}$ and $f$ on $y$ and from the boundary conditions given on $\Gamma_{\mathrm{S}}$ and $\Gamma_{\mathrm{N}}$. For example, if $C_{0}$ and $f$ are constant then

$$
Y_{n}(y)= \begin{cases}\sin \frac{n \pi y}{b}, n=1,2, \ldots, & \text { if Dirichlet B.C. (3) } \\ \cos \frac{n \pi y}{b}, n=0,1,2, \ldots, & \text { if Neumann B.C. (4) }\end{cases}
$$

It is common to refer to $n$ as the "mode number". The wave number $k$, the frequency $\omega$ and the mode number $n$ depend on each other through the dispersion relation. For example, for $C_{0}$ and $f$ constant the dispersion relation is

$$
\omega^{2}=C_{0}^{2}\left(k^{2}+\frac{n^{2} \pi^{2}}{b^{2}}\right)+f^{2}, \quad n=0,1,2, \ldots,
$$

where the mode $n=0$ appears only in the case of Neumann conditions on $\Gamma_{\mathrm{S}}$ and $\Gamma_{\mathrm{N}}$ In general, solutions of (7) consist of an infinite number of waves of the form (9). There are also solutions that decay exponentially in the $x$ direction; however, they are usually not of great concern, since the decaying modes are expected to be insignificant at the time they reach $\Gamma_{\mathrm{E}}$. Now, it is easy to verify that if one of the $C_{j}$ 's in (8) is equal to $C_{x}$, then the wave (9) satisfies the boundary condition (8) exactly.

- Each wave of the form (9) is essentially characterized by two independent parameters, say the wave number $k$ and the mode number $n$. Other parameters, like the frequency $\omega$ or the phase velocity $C_{x}$ are determined from these two parameters through the dispersion relation (see (10) and (12)). Despite this fact, the accuracy of the Higdon NRBC is determined by only one parameter per wave, namely $C_{x}$. This significantly facilitates accuracy control.

- For certain choices of the parameters, the Higdon NRBCs are equivalent to NRBCs that are derived from rational approximation of the dispersion relation (the Engquist-Majda conditions [6] being the most well-known example). This has been proved by Higdon [40,41]. More precisely, Higdon's theorem states that if a NRBC is based on a symmetric rational approximation to the dispersion relation corresponding to outgoing waves, then it is either (a) equivalent to (8) for a suitable choice of $J$ and the parameters $C_{j}$, or (b) unstable, or (c) not optimal. Lack of optimality means here that the coefficients in the $\mathrm{NRBC}$ can be modified so as to reduce the amount of the spurious reflection. Thus, the Higdon NRBCs can be viewed as generalization of rational-approximation NRBCs.

- It is easy to show (see [40] for a similar setting) that when a wave of the form (9) impinges on the boundary $\Gamma_{\mathrm{E}}$ where the NRBC $H_{J}$ is imposed, the resulting reflection coefficient is

$$
R=\prod_{j=1}^{J}\left|\frac{C_{j}-C_{x}}{C_{j}+C_{x}}\right|
$$

Again we see that if $C_{j}=C_{x}$ for any one of the $j$ 's then $R=0$, namely there is no reflection and the NRBC is exact. Moreover, we see that the reflection coefficient is a product of $J$ factors, each of which is smaller than 1. This implies that the reflection coefficient becomes smaller as the order $J$ increases regardless of the choice made for the parameters $C_{j}$. Of course, a good choice for the $C_{j}$ would lead to better accuracy with a lower order $J$, but even if we miss the correct $C_{j}$ 's considerably (say, if we make the simplest choice $C_{j}=C_{0}$ for $\left.j=1, \ldots, J\right)$, we are still guaranteed to reduce the spurious reflection as we increase the order $J$. This is an important property of Higdon's NRBCs and is the reason for their robustness.

We note that the first-order condition $H_{1}$ is a Sommerfeld-like boundary condition. If we set $C_{1}=C_{0}$ we get the classical Sommerfeld-like NRBC. A lot of work in the meteorological literature is based on using $H_{1}$ with a specially chosen $C_{1}$. Pearson [48] used a special but constant value of $C_{1}$, while in the scheme devised 
by Orlanski [49] and in later improved schemes [50-53] the $C_{1}$ changes dynamically and locally in each timestep based on the solution from the previous time-step. Some of the limited-area weather prediction codes used today are based on such schemes, e.g., COAMPS [54]. See also the recent papers [55-57] where several such adaptive $H_{1}$ schemes are compared.

Difficulties associated with the original formulation of the Higdon NRBCs (see [40]) are as follows:

- The discrete Higdon conditions were developed in the literature up to third order only, because of their algebraic complexity which increases rapidly with the order.

- The original $J$ th-order Higdon NRBC involves high normal and temporal derivatives, up to order $J$. In fact, it has the form

$$
\sum_{j=0}^{J} \gamma_{j} \partial_{x}^{j} \partial_{t}^{J-j} u=0
$$

which is obtained by expanding (8). The high derivatives pose obvious disadvantages. High normal derivatives are problematic; when finite elements are used only the $J=1$ condition is compatible with standard (low-order $C^{0}$ ) elements, whereas when finite differences are used, the discrete stencil must be a nonstandard high-order one, penetrating deeply into the computational domain away from the artificial boundary. High time derivatives are also disadvantageous in that they require the use of high-order time discretization and the storage of the solution history.

- The Higdon NRBCs involve the parameters $C_{j}$ which must be chosen. Higdon [40] discusses some general guidelines for their manual a-priori choice by the user. No procedure has been provided in the literature for the automatic choice of the $C_{j}$ 's.

The new scheme proposed in this paper overcomes all these difficulties. In the next sections we shall reformulate the Higdon NRBCs such that they can easily be used up to an arbitrarily high order. The scheme is coded once and for all for any order; the order of the scheme is simply an input parameter. Moreover, it does not involve any high derivatives. Later we shall also discuss the automatic choice of the parameters $C_{j}$.

\section{High-order non-reflecting boundary conditions}

We first replace the Higdon condition (8) by the equivalent condition

$$
H_{J}:\left[\prod_{j=1}^{J}\left(\partial_{x}+\frac{1}{C_{j}} \partial_{t}\right)\right] u=0 \quad \text { on } \Gamma_{\mathrm{E}} \text {. }
$$

Now we introduce the auxiliary functions $\phi, \ldots, \phi_{J_{1}}$, which are defined on $\Gamma_{\mathrm{E}}$ as well as in the exterior domain $D$ (see Fig. 1(b)). Eventually we shall use these functions only on $\Gamma_{\mathrm{E}}$, but the derivation requires that they be defined in $D$ as well, or at least in a non-vanishing region adjacent to $\Gamma_{\mathrm{E}}$. The functions $\phi_{j}$ are defined via the relations

$$
\begin{aligned}
& \left(\partial_{x}+\frac{1}{C_{1}} \partial_{t}\right) u=\phi_{1}, \\
& \left(\partial_{x}+\frac{1}{C_{2}} \partial_{t}\right) \phi_{1}=\phi_{2}, \\
& \vdots \\
& \left(\partial_{x}+\frac{1}{C_{J}} \partial_{t}\right) \phi_{J-1}=0 .
\end{aligned}
$$


By definition, these relations hold in $D$, and also on $\Gamma_{\mathrm{E}}$. It is easy to see that (16)-(18), when imposed as boundary conditions on $\Gamma_{\mathrm{E}}$, are equivalent to the single boundary condition (15). If we also define

$$
\phi_{0} \equiv u, \quad \phi_{J} \equiv 0,
$$

then we can write (16)-(18) concisely as

$$
\left(\partial_{x}+\frac{1}{C_{j}} \partial_{t}\right) \phi_{j-1}=\phi_{j}, \quad j=1, \ldots, J
$$

This set of conditions involves only first-order derivatives. However, due to the appearance of the $x$-derivative in (20), one cannot discretize the $\phi_{j}$ on the boundary $\Gamma_{\mathrm{E}}$ alone. Therefore we shall manipulate (20) in order to get rid of the $x$-derivative.

The function $u$ satisfies the wave equation (7) in $D$. The function $\phi_{1}$ is obtained by applying a linear operator to $u$, as in (16); hence it is clear that $\phi_{1}$ also satisfies the same equation in $D$. Similarly, we deduce that each of the functions $\phi_{j}$ satisfies a wave equation like (7), namely,

$$
\partial_{x}^{2} \phi_{j}+\partial_{y}^{2} \phi_{j}-\frac{1}{C_{0}^{2}} \partial_{t}^{2} \phi_{j}-\frac{f^{2}}{C_{0}^{2}} \phi_{j}=0 .
$$

Here we needed the assumption that $C_{0}$ and $f$ do not depend on $x$ or on $t$. Now, we make use of the following identity:

$$
\partial_{x}^{2} \phi_{j}=\left(\partial_{x}-\frac{1}{C_{j+1}} \partial_{t}\right)\left(\partial_{x}+\frac{1}{C_{j+1}} \partial_{t}\right) \phi_{j}+\frac{1}{C_{j+1}^{2}} \partial_{t}^{2} \phi_{j}
$$

Substituting (22) in (21) and replacing $j$ with $j-1$ everywhere yields, for $j=1, \ldots, J$,

$$
\left(\partial_{x}-\frac{1}{C_{j}} \partial_{t}\right)\left(\partial_{x}+\frac{1}{C_{j}} \partial_{t}\right) \phi_{j-1}+\left(\frac{1}{C_{j}^{2}}-\frac{1}{C_{0}^{2}}\right) \partial_{t}^{2} \phi_{j-1}+\partial_{y}^{2} \phi_{j-1}-\frac{f^{2}}{C_{0}^{2}} \phi_{j-1}=0 .
$$

From this and (20) we get, for $j=1, \ldots, J$,

$$
\left(\partial_{x}-\frac{1}{C_{j}} \partial_{t}\right) \phi_{j}+\left(\frac{1}{C_{j}^{2}}-\frac{1}{C_{0}^{2}}\right) \partial_{t}^{2} \phi_{j-1}+\partial_{y}^{2} \phi_{j-1}-\frac{f^{2}}{C_{0}^{2}} \phi_{j-1}=0 .
$$

On the other hand, (20) can also be written as

$$
\left(\partial_{x}+\frac{1}{C_{j+1}} \partial_{t}\right) \phi_{j}=\phi_{j+1}, \quad j=0, \ldots, J-1 .
$$

We subtract (24) from (25) to finally obtain, for $j=1, \ldots, J-1$,

$$
\left(\frac{1}{C_{j}}+\frac{1}{C_{j+1}}\right) \partial_{t} \phi_{j}=\phi_{j+1}+\left(\frac{1}{C_{j}^{2}}-\frac{1}{C_{0}^{2}}\right) \partial_{t}^{2} \phi_{j-1}+\partial_{y}^{2} \phi_{j-1}-\frac{f^{2}}{c_{0}^{2}} \phi_{j-1} .
$$

As desired, the new boundary condition (26) does not involve $x$-derivatives. In addition, there are no high $y$ - and $t$-derivatives in (26) beyond second order.

Rewriting (16), (19) and (26), we can summarize the new formulation of the $J$ th-order NRBC on $\Gamma_{\mathrm{E}}$ as follows:

$$
\beta_{0} \partial_{t} u+\partial_{x} u=\phi_{1}
$$




$$
\begin{aligned}
& \beta_{j} \partial_{t} \phi_{j}-\alpha_{j} \partial_{t}^{2} \phi_{j-1}-\partial_{y}^{2} \phi_{j-1}+\lambda \phi_{j-1}=\phi_{j+1}, \quad j=1, \ldots, J-1, \\
& \alpha_{j}=\frac{1}{C_{j}^{2}}-\frac{1}{C_{0}^{2}}, \quad \beta_{0}=\frac{1}{C_{1}}, \quad \beta_{j}=\frac{1}{C_{j}}+\frac{1}{C_{j+1}}, \quad \lambda=\frac{f^{2}}{C_{0}^{2}}, \\
& \phi_{0} \equiv u, \quad \phi_{J} \equiv 0 .
\end{aligned}
$$

\section{Finite difference discretization}

Now we consider the FD discretization of the NRBC (27)-(30). First we consider the boundary condition for $u$ (27). We discretize $\partial_{x} u$ on $\Gamma_{\mathrm{E}}$ by using the one-sided second-order approximation [58]

$$
\left(\partial_{x} u\right)_{E q}^{n} \simeq-\frac{-3 u_{E q}^{n}+4 u_{E-1, q}^{n}-u_{E-2, q}^{n} .}{2 \Delta x} .
$$

Here and elsewhere, $u_{p q}^{n}$ (and also $u_{p, q}^{n}$ ) is the FD approximation of $u(x, y, t)$ at grid point $\left(x_{p}, y_{q}\right)$ and at time $t_{n}$, and the index $E$ correspond to a grid point on the boundary $\Gamma_{\mathrm{E}}$. From (27) we obtain a discrete formula for $\partial_{t} u$, i.e.,

$$
\left(\partial_{t} u\right)_{E q}^{n} \simeq \frac{1}{\beta_{0}}\left(\left(\phi_{1}\right)_{E q}^{n}-\left(\partial_{x} u\right)_{E q}^{n}\right) .
$$

Then we calculate the new $u$ by a forward-in-time difference formula. We can either use the two-level firstorder formula

$$
u_{E q}^{n+1}=u_{E q}^{n}+\Delta t\left(\partial_{t} u\right)_{E q}^{n}
$$

or the three-level second-order formula [58]

$$
u_{E q}^{n+1}=\frac{1}{3}\left(4 u_{E q}^{n}-u_{E q}^{n-1}+2 \Delta t\left(\partial_{t} u\right)_{E q}^{n}\right) .
$$

In obtaining the numerical examples presented in Section 7 we have chosen to work with the first-order formula (33). It should be noted that when one uses a high-order NRBC (namely $H_{J}$ with a large $J$ ), the discrete operator involved effectively for $u$ on the boundary is of high-order even when a first-order formula like (33) is used. Thus the importance of using boundary discretization schemes with improved accuracy diminishes when $J$ increases.

Next we consider the boundary condition for $\phi_{j}(28)$. We use the following second-order central difference approximations for the second temporal and tangential derivatives [58]:

$$
\begin{aligned}
&\left(\partial_{t}^{2} \phi_{j-1}\right)_{E q}^{n} \simeq \frac{\left(\phi_{j-1}\right)_{E q}^{n+1}-2\left(\phi_{j-1}\right)_{E q}^{n}+\left(\phi_{j-1}\right)_{E q}^{n-1}}{(\Delta t)^{2}}, \\
&\left(\partial_{y}^{2} \phi_{j-1}\right)_{E q}^{n+1} \simeq \frac{\left(\phi_{j-1}\right)_{E, q+1}^{n+1}-2\left(\phi_{j-1}\right)_{E q}^{n+1}+\left(\phi_{j-1}\right)_{E, q-1}^{n+1}}{(\Delta y)^{2}} .
\end{aligned}
$$

Note that (36) cannot be used at the two east corners (the two end points of $\Gamma_{\mathrm{E}}$ ). At these corners, we do one of the following things. If the Dirichlet boundary conditions (3) are applied on $\Gamma_{\mathrm{S}}$ and $\Gamma_{\mathrm{N}}$, then it is 
easy to show from (16)-(18) that on these boundaries not only $u=0$ but also $\phi_{j}=0$ for $j=1, \ldots, J$. Thus, in the two east corners we simply take $u=0$ and $\phi_{j}=0$ for all the $j$ s. If the Neumann boundary conditions (4) are given on $\Gamma_{\mathrm{S}}$ and $\Gamma_{\mathrm{N}}$, we use at the two east corners a one-sided second-order approximation instead of the central-difference formula (36). For example, at the south-east corner we use [58]

$$
\left(\partial_{y}^{2} \phi_{j-1}\right)_{E q}^{n+1} \simeq \frac{2\left(\phi_{j-1}\right)_{E q}^{n+1}-5\left(\phi_{j-1}\right)_{E, q+1}^{n+1}+4\left(\phi_{j-1}\right)_{E, q+2}^{n+1}-\left(\phi_{j-1}\right)_{E, q+3}^{n+1}}{(\Delta y)^{2}}
$$

From (28) we obtain a discrete formula for $\partial_{t} \phi_{j}$, i.e.,

$$
\left(\partial_{t} \phi_{j}\right)_{E q}^{n} \simeq \frac{1}{\beta_{j}}\left(\left(\phi_{j+1}\right)_{E, q+1}^{n}+\alpha_{j}\left(\partial_{t}^{2} \phi_{j-1}\right)_{E q}^{n}+\left(\partial_{y}^{2} \phi_{j-1}\right)_{E q}^{n+1}-\lambda\left(\phi_{j-1}\right)_{E q}^{n+1}\right) .
$$

Then we calculate the new $\phi_{j}$ by a forward-in-time formula, which again can be either the two-level firstorder formula

$$
\left(\phi_{j}\right)_{E q}^{n+1}=\left(\phi_{j}\right)_{E q}^{n}+\Delta t\left(\partial_{t} \phi_{j}\right)_{E q}^{n}
$$

(which we actually use in Section 7) or the three-level second-order formula

$$
\left(\phi_{j}\right)_{E q}^{n+1}=\frac{1}{3}\left(4\left(\phi_{j}\right)_{E q}^{n}-\left(\phi_{j}\right)_{E q}^{n-1}+2 \Delta t\left(\partial_{t} \phi_{j}\right)_{E q}^{n}\right) .
$$

The simplest solution procedure is the one based on the sequential solution of the equations for the $\phi_{j}$ 's. Namely, we first solve for $u$, then we solve for $\phi_{1}$, then for $\phi_{2}$, and so on. At the stage when we update the values of $\phi_{j}$, the quantities $\left(\phi_{j-1}\right)_{E . .}^{n+1}$ appearing in (35) and (36) are already known, having been derived in the previous stage for $\phi_{j-1}$. On the other hand, the quantity $\left(\phi_{j+1}\right)_{E q}^{n+1}$ is not yet available; that is why we use $\left(\phi_{j+1}\right)_{E q}^{n}$ in (32) and (38) rather than $\left(\phi_{j+1}\right)_{E q}^{n+1}$. The latter fact may potentially lead to an unstable solution. Indeed, when we have implemented the scheme based on the formulas (31)-(39) with $J \geqslant 2$, an instability developed in time. One remedy for this instability is to perform a second iteration and update $u$ and the $\phi_{j}$ 's again based on values obtained in the first iteration. This two-cycle algorithm turns out to be stable. It is summarized in Box 1.

Note that the only algorithmic difference between the first and second iterations is in the use of $\left(\phi_{1}\right)_{E q}^{n}$ vs. $\left(\phi_{1}\right)_{E q}^{n+1}$ in (32). All the other formulas remain unchanged in the two iterations. We have tried to use also $\left(\phi_{j+1}\right)_{E q}^{n+1}$ in (38) instead of $\left(\phi_{j+1}\right)_{E q}^{n}$ in the second iteration, but this led to instability.

As an alternative scheme, Eqs. (31)-(39) may be solved simultaneously for all the $j$ 's and all the $y$-locations $q$, as one coupled system of linear equations on $\Gamma_{\mathrm{E}}$. The dimension of this system is $J N_{y}$, where $N_{y}$ is the number of grid-points on $\Gamma_{\mathrm{E}}$ However, in the numerical examples presented in Section 7 we have used the decoupled discretization scheme described in Box 1.

In the interior domain $\Omega$, we use the standard second-order centered difference scheme

$$
u_{p q}^{n+1}=2 u_{p q}^{n}-u_{p q}^{n-1}+\left(\frac{C_{0} \Delta t}{\Delta x}\right)^{2}\left(u_{p+1, q}^{n}-2 u_{p q}^{n}+u_{p-1, q}^{n}\right)+\left(\frac{C_{0} \Delta t}{\Delta y}\right)^{2}\left(u_{p, q+1}^{n}-2 u_{p q}^{n}+u_{p, q-1}^{n}\right)-(f \Delta t)^{2} u_{p q}^{n} .
$$

Higdon [40] has proved that the discrete NRBCs (8) (in their original form) are stable if (41) is used as the interior scheme. Since both (41) and the discretized NRBC are explicit, the whole scheme is explicit. 


\section{Computational issues}

\subsection{Complexity}

From Eqs. (31)-(39) and Box 1, it is easy to see that the number of operations related to the $J$ th-order $\mathrm{NRBC}$ on $\Gamma_{\mathrm{E}}$ is $\mathrm{O}\left(J N_{y}\right)$ per time-step, where $N_{y}$ is the number of grid-points on $\Gamma_{\mathrm{E}}$. The associated computational effort is typically marginal with respect to the total effort required by the entire solution process. In comparison, the scheme proposed in [46] which directly uses the original Higdon NRBCs (but with a special high-order discretization scheme) requires $\mathrm{O}\left(3^{J} N_{y}\right)$ operations per time-step, namely its complexity grows exponentially with $J$.

- First iteration:

- Compute the $\left(\partial_{x} u\right)^{n}$ values on $\Gamma_{\mathrm{E}}$ from (31).

- Compute the $\left(\partial_{t} u\right)^{n}$ values on $\Gamma_{\mathrm{E}}$ from (32).

- Compute the $u^{n+1}$ values on $\Gamma_{\mathrm{E}}$ from (33).

- If $J=1$, stop.

- For $j=1, \ldots, J-1$ :

- Compute the $\partial_{t}^{2}\left(\phi_{j-1}\right)^{n}$ values from (35).

- Compute the $\partial_{y}^{2}\left(\phi_{j-1}\right)^{n+1}$ values from (36) and (in the two corners) from (37).

- Compute the $\partial_{t}\left(\phi_{j}\right)^{n}$ values from (38).

- Compute the $\left(\phi_{j}\right)^{n+1}$ values from (39).

- Next $j$

- Second iteration:

- Recompute the $\left(\partial_{t} u\right)^{n}$ values on $\Gamma_{\mathrm{E}}$ from (32), but use $\left(\phi_{1}\right)^{n+1}$ instead of $\left(\phi_{1}\right)^{n}$.

- Recompute the $u^{n+1}$ values on $\Gamma_{\mathrm{E}}$ from (33).

- For $j=1, \ldots, J-1$ :

- Recompute the $\partial_{t}^{2}\left(\phi_{j-1}\right)^{n}$ values from (35).

- Recompute the $\partial_{y}^{2}\left(\phi_{j-1}\right)^{n+1}$ values from (36) and (in the two corners) from (37).

- Recompute the $\partial_{t}\left(\phi_{j}\right)^{n}$ values from (38).

- Recompute the $\left(\phi_{j}\right)^{n+1}$ values from (39).

- Next $j$

Box 1. Algorithm for the FD implementation of the $J$ th-order NRBC.

\subsection{Choosing the parameters $C_{j}$}

Now we discuss how to choose the $J$ parameters $C_{j}$, appearing in the NRBC (27)-(30).

First, we note that some physical limitations may apply to the chosen values of the $C_{j}$ 's. For example, if $C_{0}$ and $f$ are constant then from (10) and (12) we get

$$
C_{x}=C_{0}=\sqrt{1+\frac{n^{2} \pi^{2} / b^{2}+f^{2}}{k^{2}}}, \quad n=0,1,2, \ldots
$$

and thus $C_{x} \geqslant C_{0}$. Hence in this case one should take $C_{j} \geqslant C_{0}$ for all the $j$ 's.

Second, we recall that even the simple choice $C_{j}=C_{0}$ for $j=1, \ldots, J$, is guaranteed to reduce the spurious reflection as $J$ increases (see Section 2). Thus, this choice may be successful in many cases provided that $J$ is sufficiently large. What one gains from making a specialized choice for the $C_{j}$ is the ability to obtain 
the desired level of accuracy with a smaller order $J$. However, one must bear in mind that since the NRBCassociated cost increases only linearly with $J$, taking a large $J$ may sometimes be preferable to spending a considerable amount of computational effort on a sophisticated procedure to choose the $C_{j}$.

Three approaches for choosing the parameters $C_{j}$ suggest themselves:

(a) The user chooses the $C_{j}$ a priori in a manual manner based on an "educated guess."

(b) The $C_{j}$ are chosen automatically by the computer code as a preprocess.

(c) The $C_{j}$ are not constant, but are determined dynamically by the computer code.

Approach (a) is the one recommended in Higdon's papers [40-45]. It is based on the assumption that the user has some a priori knowledge on the character of the exact solution. While this may be a good assumption in some applications, it is definitely desirable to have at hand an automatic procedure that will not require the user's intervention.

Approach (b) is attractive since it is automatic yet very inexpensive computationally. We have adopted this approach in [46] as well as here, using an algorithm which is based on the maximum resolvable wave numbers in the $x$ and $y$ directions, and on the minimax formula [59] for choosing the $x$ component wave numbers. The algorithm consists of the following steps (see [46] for further details): (1) Estimate the maximum resolvable wave number $k$ in the $x$ direction. Assuming a maximum of 10 grid points per wavelength, a reasonable estimate is $k_{\max }=\pi /(5 \Delta x)$, where $\Delta x$ is the grid spacing in the $x$ direction. (2) Similarly, estimate the maximum resolvable wave number $k$ in the $y$ direction. (3) Choose $J$ values of $k$ from the interval $\left(0, k_{\max }\right]$. The simplest choice is the uniform division, namely $k_{j}=k_{\max } j / J$. However, a better choice is to take the roots of the Chebyshev polynomial as the $k_{j}$. This choice is based on the symmetric minimax formula, proposed by Sommeijer et al. [59]. In this case more of the $k_{j}$ are concentrated near the ends of the interval $\left(0, k_{\max }\right]$. (4) For each $k_{j}$, calculate the corresponding (and maximal in the $y$ direction) frequency $\omega_{j}$ from the dispersion relation (cf. (12)). (5) Calculate $C_{j}=\omega_{j} / k_{j}$ (see (10)).

Approach (c) is the most sophisticated, and also the most expensive computationally. In this approach, the values $C_{j}$ are estimated for every grid point on the boundary at each time-step, from the solution in the previous time-steps. For the Sommerfeld-like NRBC $(J=1)$, a procedure of this type is used a lot in meteorological applications [49-53]. Analogous procedures may be employed with higher orders, although Higdon [40] reports bad results with the Orlanski scheme [49] with $J=2$ and $J=3$. An adaptive scheme of a different type, perhaps more suitable to high orders, is based on the fast Fourier transform (FFT); see [60].

\subsection{Exactness of the scheme}

The proposed high-order NRBC scheme is converging, in the following sense. Consider the exact solution of two problems: the first is the original problem in the infinite domain, and the second is the problem in the truncated domain $\Omega$, with the NRBC applied on $\mathscr{B}$. Then the distance $\delta$, in some reasonable norm, between the two solutions in $\Omega$ may serve as an error measure. Now, if a sequence of NRBCs satisfies the following two properties:

(1) for any given problem of the class under consideration the error measure $\delta$ of the NRBC approaches zero as the order of the NRBC $J$ goes to infinity while $\mathscr{B}$ is held fixed;

(2) the NRBC can be implemented once and for all for any order $J$, without limit;

then it is justified to call the sequence of NRBCs exact, or converging. In this sense the Grote-Keller NRBC [15], the three-dimensional Hagstrom-Hariharan NRBC [19] and the localized DtN condition [22] are all converging.

The NRBC scheme proposed in the present paper is also converging. The reason is that by increasing $J$ and choosing the parameters $C_{J}$ in an appropriate way, one is assured to reduce the spurious reflection down to any desired level. To demonstrate this we give two arguments: a theoretical one and a practical one. 
The theoretical argument is as follows. Consider the case of constant $C_{0}$ and $f$. Any solution of (7) may be written as a combination of plane waves, each one associated with a certain phase velocity in the $x$ direction, $C_{x} \geqslant C_{0}$ (see (9)-(12)). Each wave generates the reflection coefficient $R$ given by (13), which is a product of numbers smaller than one. Thus, as $J$ increases, the $R$ generated by each wave must decrease. However, this does not necessarily mean that $R$ approaches zero as $J \rightarrow \infty$. A sufficient condition for this to be guaranteed is that the set of $C_{j}$ 's is dense in the interval $\left[C_{0}, \infty\right)$ as $J \rightarrow \infty$, so that whatever $C_{x}$ is, it will be arbitrarily close to one of the $C_{j}$. This is indeed possible to achieve: the sequence of $C_{j}$ 's may be taken to be the sequence of all the rational numbers greater than or equal to $C_{0}$. The rational numbers form a countable set and thus can be ordered in a sequence; moreover they are dense in the set of real positive numbers (a non-countable set). This argument is "theoretical" because in practice the $C_{j}$ will never be chosen in this fashion.

The practical argument is as follows. It is true that the phase velocity $C_{x}$ of the waves participating in the solution is not necessarily bounded, namely that for any number $M$ there may be waves for which $C_{x}>M$. However, those waves that have very high $C_{x}$ are not of practical interest, since they cannot be resolved by the discrete scheme anyway. Moreover, such waves are expected to have small amplitudes and not to influence the solution significantly compared to the resolved waves (otherwise the discretization is not satisfactory). Thus, we can estimate a "practical bound" $\left(C_{x}\right)_{\max }$ for the $C_{x}$ (say, a multiple of $\Delta x / \Delta t$ ). Then we only have to consider the finite interval $C_{j} \in\left[C_{0},\left(C_{x}\right)_{\max }\right]$. The $C_{j}$ may be chosen by a uniform division of this interval into $J$ values, or by using the algorithm outlined in Section 5.2. Either way, as $J \rightarrow \infty$, the set of the $C_{j}$ 's is dense in this interval.

\subsection{Stability}

Since the proposed method is explicit in time, one has to be concerned about numerical stability. For stability of the interior scheme (41), we must satisfy the well-known CFL condition $C_{0} \Delta t / \Delta x \leqslant 1$. Since typically one would choose $\Delta t / \Delta x$ to be slightly below the stability limit, we assume that $\Delta t$ and $\Delta x$ are chosen such that $C_{0} \Delta t / \Delta x=1-\epsilon$. Now, it is expected that the NRBC will also be associated with a stability condition. Indeed, the condition (27) and its discrete counterpart (31)-(33) point to the CFL condition $\left(1 / \beta_{0}\right) \Delta t / \Delta x \leqslant 1$. We thus conclude that for stability,

$$
\beta_{0} \equiv \frac{1}{C_{1}} \geqslant \frac{\Delta t}{\Delta x}=\frac{1-\epsilon}{C_{0}}
$$

A similar argument, as well as numerical experiments, lead also to the sufficient condition for stability

$$
\beta_{j} \equiv \frac{1}{C_{j}}+\frac{1}{C_{j+1}} \geqslant \frac{1}{C_{0}}
$$

for $j=1, \ldots, J-1$. Satisfying (43) and (44) is usually easy if one arranges the parameters $C_{j}$ in an appropriate way. For example, for $J=4$, the arrangement $C_{j} / C_{0}=1,4,4,1$ is unstable (since $\left.\beta_{2}=1 /\left(2 C_{0}\right)<1 / C_{0}\right)$ but $C_{j} / C_{0}=1,4,1,4$ is stable. If a general automatic procedure is used to choose the $C_{j}$, then this procedure should take into account the stability limitations. This can be done, for example, by sorting the $C_{j}$ appropriately and by replacing some of them by the reference phase speed $C_{0}$. Of course, the issue of the NRBC stability does not arise at all if Eqs. (27)-(30) on $\Gamma_{\mathrm{E}}$ are solved in an implicit manner.

\section{Numerical examples}

We apply the new scheme to a number of test problems in a wave guide, as described in Section 2 and illustrated in Fig. 1(b). We set $b=5$ and $C_{0}=1$. The initial conditions are zero throughout the domain. 
On the walls $\Gamma_{\mathrm{S}}$ and $\Gamma_{\mathrm{N}}$ we use the Dirichlet boundary condition (3). We consider two cases: the nondispersive case, namely $f=0$, and the dispersive case, with $f=0.5$. On the boundary $\Gamma_{\mathrm{W}}$ we take the Dirichlet boundary condition (5), with

$$
u_{\mathrm{W}}(y, t)=\sin \left(\frac{\pi y}{b}\right) \cos k C_{x} t, \quad t>0 .
$$

This has the form of a mode- 1 wave (see (9)). In (45) we take $k=0.1$, and compute from the dispersion relation (12) the phase velocity $C_{x}=6.3623$ for the case $f=0$ and $C_{x}=8.0966$ for the case $f=0.5$. Note that the sine-wave "loading" (45) is activated at $t=0$ in a step-like fashion, since for $t<0$ the solution is zero.

We introduce the artificial boundary $\Gamma_{\mathrm{E}}$ (see Fig. 1(b)) at $x_{\mathrm{E}}=5$. Thus, the computational domain $\Omega$ is a $5 \times 5$ square. In $\Omega$ we use a uniform grid with $21 \times 21$ points. We discretize the Klein-Gordon equation in $\Omega$ using the explicit central-difference FD interior scheme (41). The time-step size is $\Delta t=0.025$, which is smaller than the CFL stability limit. On $\Gamma_{\mathrm{E}}$ we impose the new NRBC, while varying the order $J$. Initially we use $C_{j}=1$ for all the $j$ 's.

A reference solution which is regarded as the "exact solution" $u_{\mathrm{ex}}$ is obtained by solving the problem in a longer domain, with a refined grid and with a very high-order NRBC on the artificial boundary. Fig. 2 shows the "exact solution" obtained for the case $f=0.5$ by performing 4000 time-steps. The solution is shown at the point $P(5,2.75)$ (corresponding to the grid point $p=21, q=12$ ) which is located on the artificial boundary $\Gamma_{\mathrm{E}}$, slightly above the center of the waveguide. As the graph shows, the solution is initially zero, until the first noticeable wave packet reaches this point. Then there is a strong dynamic response which after a short time decays rapidly.

To demonstrate the difficulties involved in this basic example, we consider the angle of incidence $\theta$ of the incoming wave at the point $P(5,2.75)$ on $\Gamma_{\mathrm{E}}$. This is defined as the angle between the wave direction $\gamma$ and the normal to $\Gamma_{\mathrm{E}}$ at $P$, namely $\theta=\left|90^{\circ}-\gamma\right|$. The wave direction $\gamma$ is computed via

$$
\cos \gamma=\frac{\nabla u}{|\nabla u|} \cdot \boldsymbol{e}_{y}=\frac{\partial_{y} u}{\sqrt{\left(\partial_{x} u\right)^{2}+\left(\partial_{x} u\right)^{2}}},
$$

where $\gamma$ is measured clockwise from the $-y$ axis and $\boldsymbol{e}_{y}$ is the unit vector pointing in the $y$ direction. The partial derivatives appearing in (46) can be calculated via the expressions

$$
\partial_{x} u=-k \sin \left(\frac{\pi y}{b}\right) \sin k\left(x-C_{x} t\right), \quad \partial_{y} u=(\pi / b) \cos \left(\frac{\pi y}{b}\right) \cos k\left(x-C_{x} t\right),
$$

which correspond to the mode-1 wave

$$
u=\sin \left(\frac{\pi y}{b}\right) \cos k\left(x-C_{x} t\right)
$$

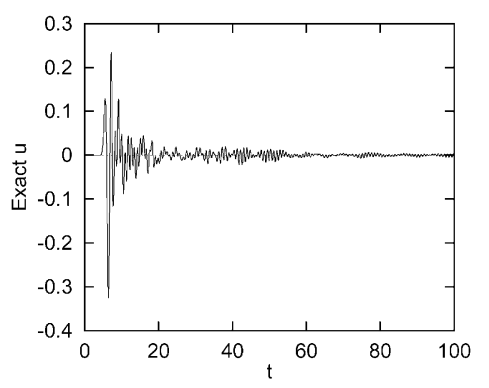

Fig. 2. Exact solution for the wave-guide test problem, in the dispersive case $(f=0.5)$. The solution is shown at the point $P(5,2.75)$. 

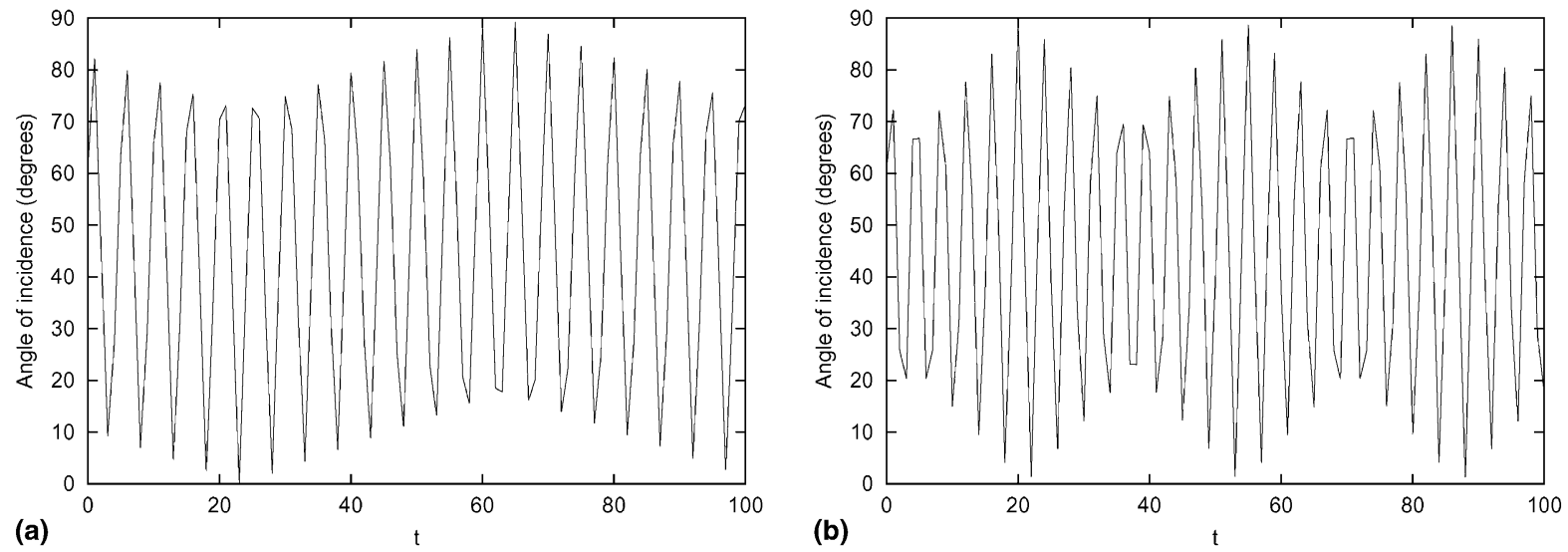

Fig. 3. Angle of incidence at the point $P(5,2.75)$ on the artificial boundary as a function of time: (a) in the non-dispersive case $(f=0)$, and (b) in the dispersive case $(f=0.5)$.

where transient effects due to the step-in-time character of the wave are neglected. Fig. 3 shows the angle of incidence $\theta$ at $P$ as a function of time for the non-dispersive and dispersive cases. In both cases, $\theta$ varies between $0^{\circ}$ and $90^{\circ}$ in an oscillatory manner. A different example in which the angle of incidence is changing (in fact, increasing) in time was given by Hagstrom [5]. Such cases where there are waves with a wide range of incidence angles are known to be relatively difficult for NRBC treatment.

We define two error measures. The first one is the Eulerian norm of the error over $\Gamma_{\mathrm{E}}$, i.e.,

$$
E(t)=\sqrt{\frac{1}{N_{y}} \sum_{m=1}^{N_{y}}\left(u\left(x_{\mathrm{E}}, y_{m}, t\right)-u_{\mathrm{ex}}\left(x_{\mathrm{E}}, y_{m}, t\right)\right)^{2}} .
$$

The second measure is the pointwise error at a single point $\left(x_{\mathrm{E}}, y_{0}\right) \Gamma_{\mathrm{E}}$, i.e.,

$$
e(t)=u\left(x_{\mathrm{E}}, y_{0}, t\right)-u_{\mathrm{ex}}\left(x_{\mathrm{E}}, y_{0}, t\right) .
$$

Fig. 4 shows the errors generated by the new scheme with $J=3$. In Fig. 4(a) the global error $E$ defined by (49) is plotted, while Fig. 4(b) corresponds to the pointwise error $e$ defined by (50), at the point $P$ mentioned above. It is apparent that the errors are oscillatory. Their amplitudes grow initially, reach a maximum, and then decay in time, albeit more slowly than the solution itself. Note that the frequency of the error oscillation is similar to that of the angle of incidence of the waves at the point $P$ on $\Gamma_{\mathrm{E}}$ as seen in Fig. 3. This is not surprising since the accuracy of the Higdon conditions is known to be strongly affected by the angle of incidence (see [41,42]).

In Figs. 5 and 6 we compare the numerical errors for different orders $J$, calculated in the first 600 timesteps. The figures show the global errors (Fig. 5) and pointwise errors (Fig. 6) as a function of time for the non-dispersive $(f=0)$ and dispersive $(f=0.5)$ cases. In each case we show the error generated by the new NRBC of order $J=1,3,5$, and 7. It is apparent that the errors decrease as the order $J$ increases. We also see that for $J \geqslant 2$ the error generated in the dispersive case is larger than that in the non-dispersive case. Note that auxiliary variables appear in the formulation only for $J \geqslant 2$, so the case $J=1$ is special.

Now we consider the same wave guide as in the previous example, but with a different loading. On the west boundary we replace (45) by the prescribed function

$$
u_{\mathrm{W}}(y, t)= \begin{cases}\cos \left[\frac{\pi}{2 r_{s}}\left(y-y_{s}\right)\right] & \text { if }\left|y-y_{s}\right| \leqslant r_{s} \text { and } 0<t \leqslant t_{s}, \\ 0 & \text { otherwise. }\end{cases}
$$



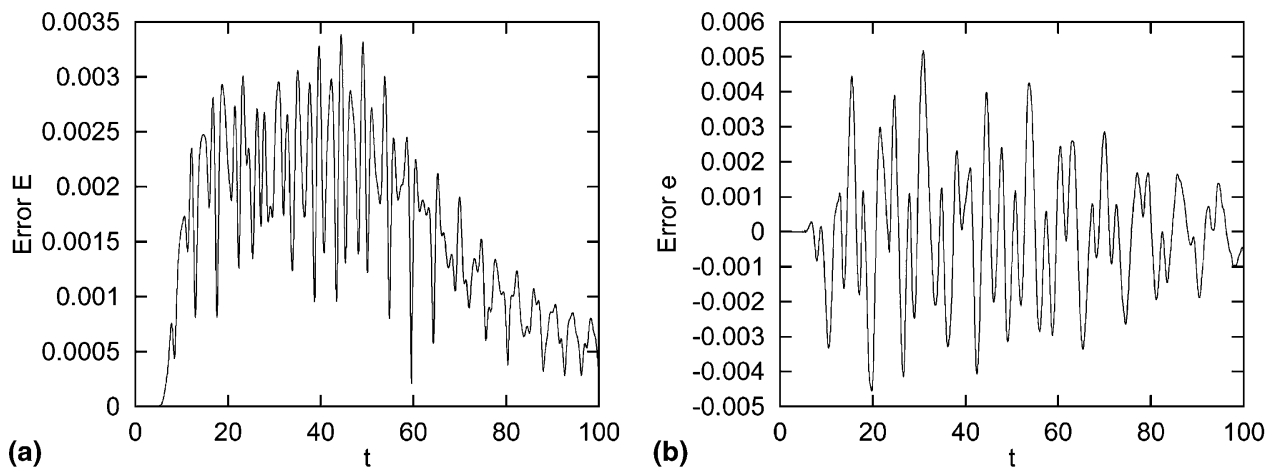

Fig. 4. Errors as a function of time, for the dispersive case $(f=0.5)$, generated by the new NRBC of order $J=3$ : (a) the global error $E$ defined by (49); (b) the pointwise error $e$ defined by (50), at the point $P(5,2.75)$.
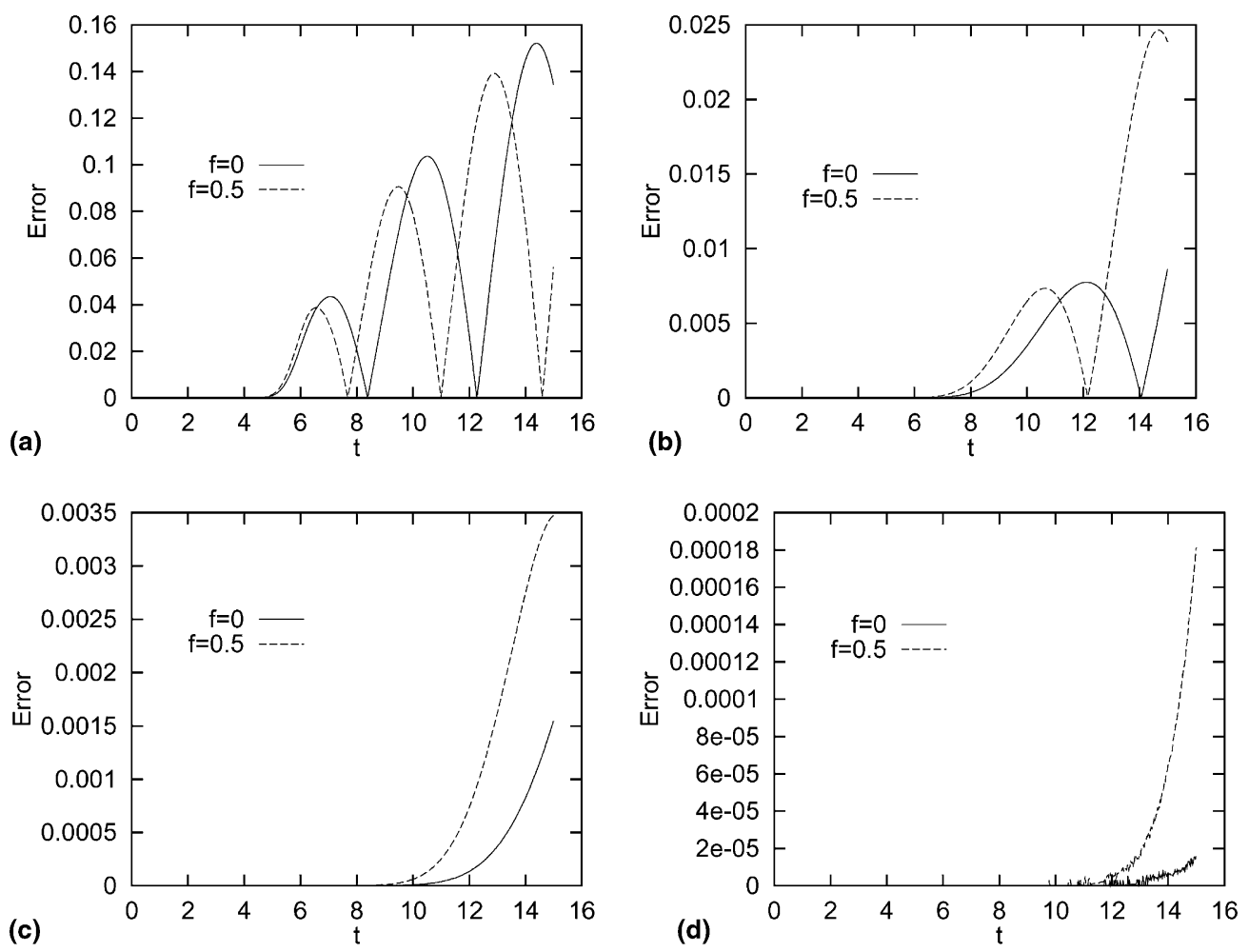

Fig. 5. Errors calculated by the global measure $E$ defined by (49), as a function of time, for the non-dispersive $(f=0)$ and dispersive $(f=0.5)$ cases. The new NRBC of order $J$ is used on $\Gamma_{\mathrm{E}}$ : (a) $J=1$, (b) $J=3$, (c) $J=5$, and (d) $J=7$.

Thus, the wave source on the west boundary is a cosine function in $y$ with three parameters: its center location $y_{s}$, its width $r_{s}$, and its time duration $t_{s}$. We set $y_{s}=1, r_{s}=1$, and $t_{s}=0.5$. We take $f=0.5$ (the dispersive case). The computational parameters $x_{\mathrm{E}}, \Delta t$ and the grid are the same as in the previous example. However, here we use the NRBC of order $J=4$ on $\Gamma_{\mathrm{E}}$, with the four $C_{j}$ 's obtained automatically by 

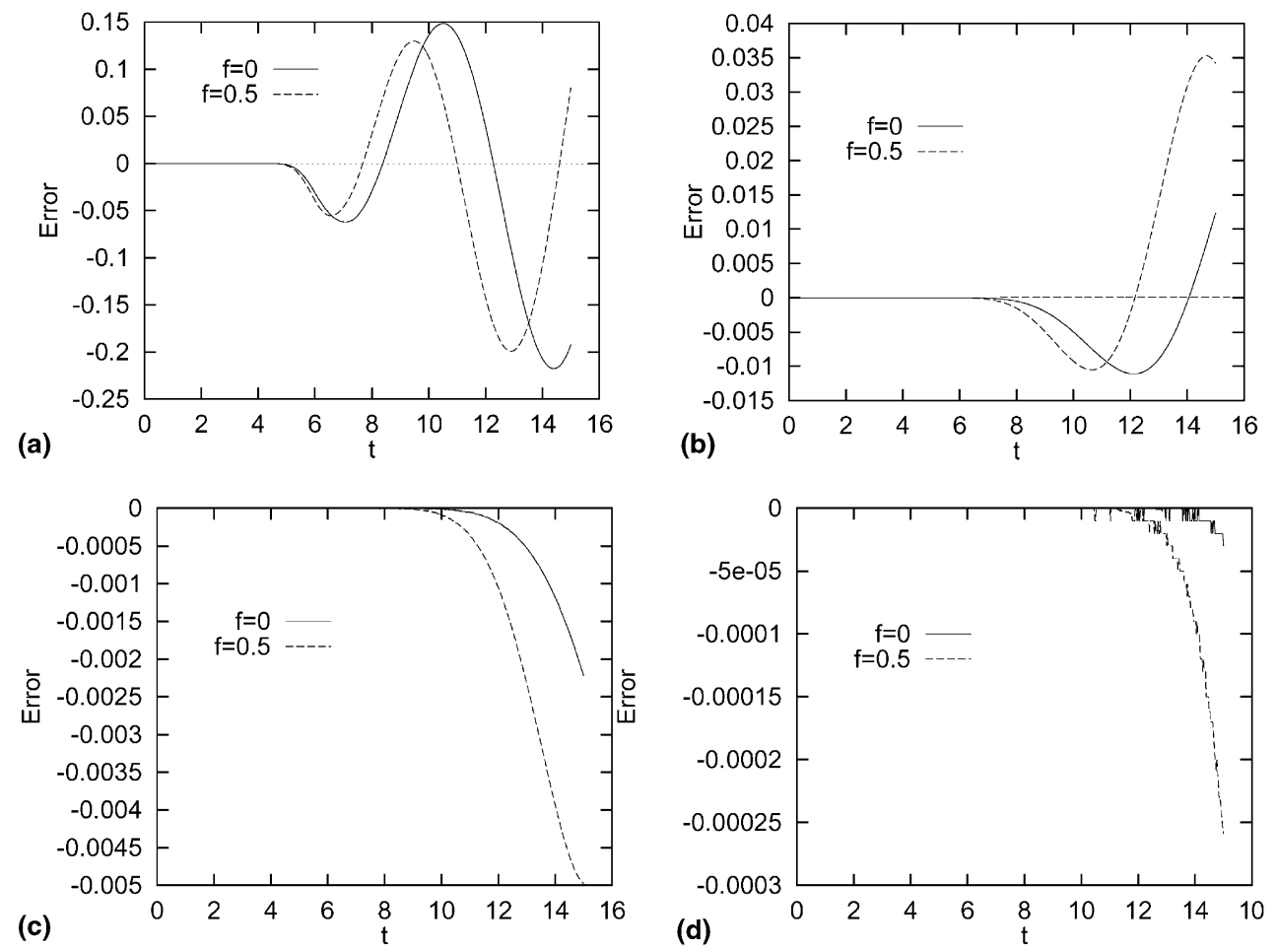

Fig. 6. Errors calculated by the pointwise measure $e$ defined by (50), as a function of time, for the non-dispersive $(f=0)$ and dispersive $(f=0.5)$ cases. The new NRBC of order $J$ is used on $\Gamma_{\mathrm{E}}$ : (a) $J=1$, (b) $J=3$, (c) $J=5$, and (d) $J=7$.

employing the procedure outlined in Section 5.2. These turn out to be $C_{j} / C_{0}=1,1.45,1.75,4.06$. For stability reasons (see Section 5.4) we replace these values with $C_{j} / C_{0}=1,1.45,1,4.06$.

We compare the solution obtained by the new scheme with two other solutions:

- A solution obtained in the same domain, but with the Higdon NRBC $H_{1}$ on $\Gamma_{\mathrm{E}}$ using $C_{1}=5$. (Results obtained for $H_{1}$ with a smaller $C_{1}$ had a similar character, although less dramatic quantitatively.)

- A solution obtained in a domain twice as long, namely the domain $0 \leqslant x \leqslant 10,0 \leqslant y \leqslant 5$, using a $42 \times 21$ grid with the same resolution. During the simulation time the wave generated on $\Gamma_{\mathrm{W}}$ does not reach the remote (east) boundary of this large domain, and thus the issue of spurious reflection is avoided altogether, regardless of the boundary condition used on the remote boundary. Hence this will serve as a reference solution.

Fig. 7(a) shows the three solutions at time $t=0.5$, which occurs exactly when the wave source is "turned off." In this and the next figures, the top plot is that of the reference solution, the middle plot corresponds to the solution obtained by the new scheme with the fourth-order NRBC, and the lower plot describes the $H_{1}$ solution. Both the colors and the contour lines represent values of $u$. At time $t=0.5$ all three solutions are identical, as expected. The largest contour line shown represents a fast wave with a small amplitude which is present due to the dispersion.

Fig. 7(b) corresponds to time $t=6$. At this time the main bulk of the wave packet generated on $\Gamma_{\mathrm{W}}$ has just reached the boundary $\Gamma_{\mathrm{E}}$. The three plots are still similar, although a slight spurious reflection near the boundary can be observed in the $H_{1}$ solution.

Fig. 7(c) shows the three solutions at time $t=7.5$. The main wave packet has already passed the boundary $\Gamma_{\mathrm{E}}$. The fourth-order solution is almost indistinguishable from the reference solution, whereas in 

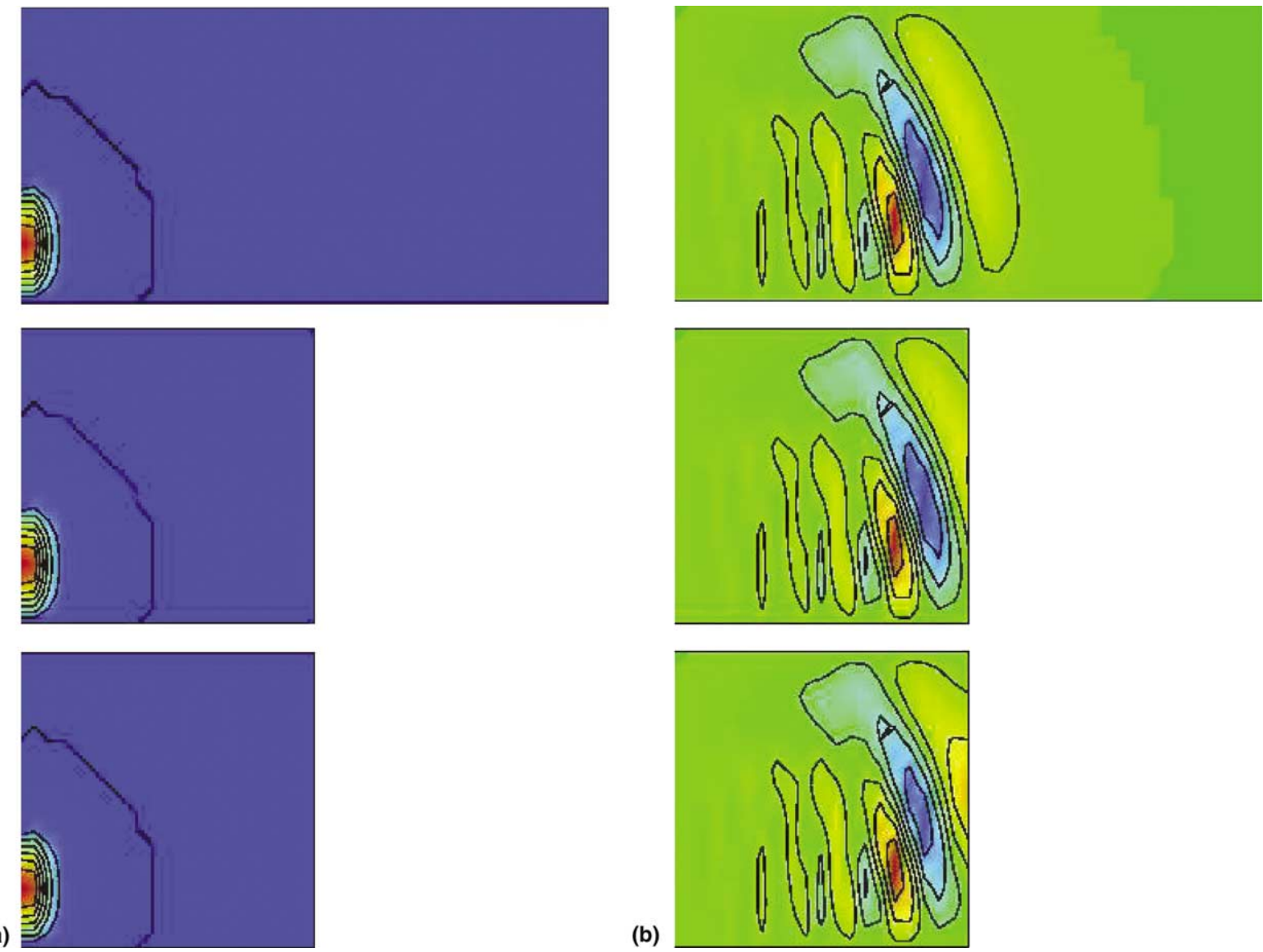

(b)

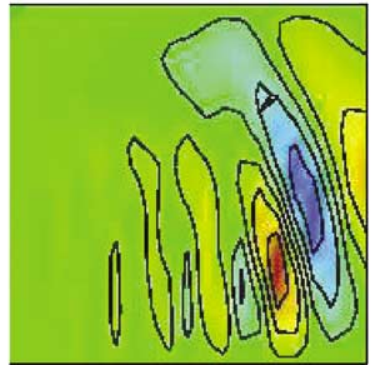

Fig. 7. Solution of the cosine-source problem, dispersion parameter $f=0.5$. Top plot - reference solution in a long domain. Middle plot-solution obtained with the new scheme, fourth-order NRBC. Lower plot-solution obtained with the Sommerfeld-like NRBC $H_{1}$. Solutions are shown at times: (a) $t=0.5$, (b) $t=6$, (c) $t=7.5$, (d) $t=9.5$, and (e) $t=11$.

the $H_{1}$ solution spurious reflection is evident. Figs. 7(d) and (e) correspond to times $t=9.5$ and $t=11$, respectively. The reflected waves move backwards in the $H_{1}$ solution and pollute the entire computational domain. On the other hand, the fourth-order solution exhibits, with a reasonable accuracy, the wave traces which are also present in the reference solution.

In order to examine the errors generated in this example quantitatively, we introduce the new global error measure $E_{\Omega}(t)$, defined by

$$
E_{\Omega}(t)=\sqrt{\frac{1}{N_{x} N_{y}} \sum_{p=1}^{N_{x}} \sum_{q=1}^{N_{y}}\left(u\left(x_{p}, y_{q}, t\right)-u_{\mathrm{ex}}\left(x_{p}, y_{q}, t\right)\right)^{2} .}
$$

Here $N_{x}$ and $N_{y}$ are, respectively, the number of grid points in the $x$ and $y$ directions in the computational domain $\Omega$. In traveling-pulse type problems like the present example, the error measure $E_{\Omega}(t)$ is preferred over $E$ defined in (49) or $e$ defined in (50). The reason is that the latter measures indicate the error only over the boundary $\Gamma_{\mathrm{E}}$, while, as Figs. 7(a)-(e) demonstrate, the major errors are not necessarily found on this boundary but they penetrate the interior and constantly change their locations. 

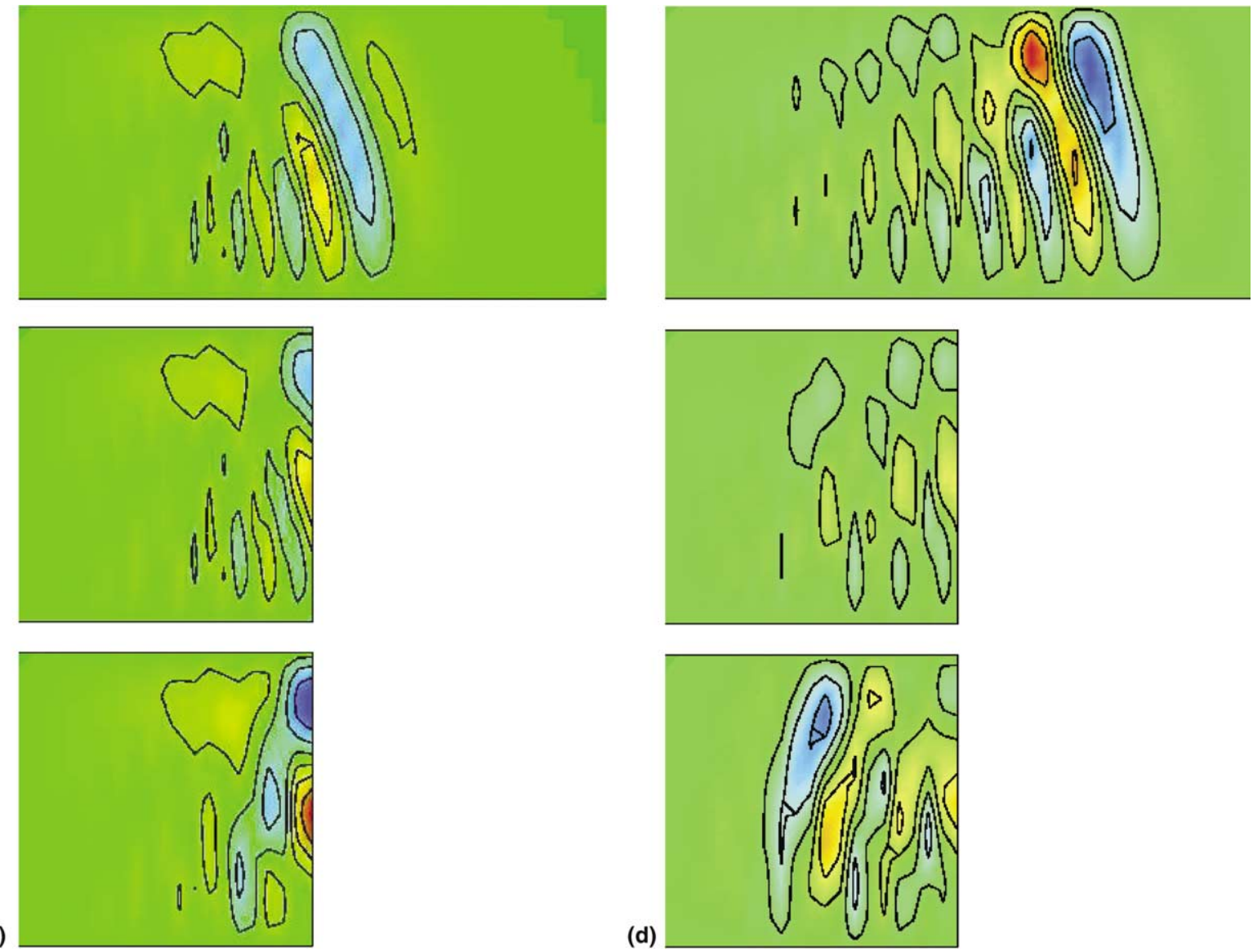

(d)

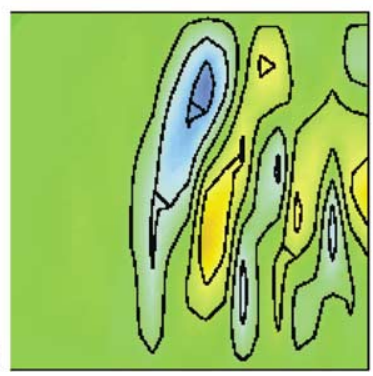

Fig. 7. (continued)

Fig. 8 shows the global error $E_{\Omega}$ as a function of time for the $J=4$ solution and for the $J=1$ solution. $\mathrm{Up}$ until $t=10$, before the wave reaches the artificial boundary, both errors are extremely small. Once the wave reaches the boundary both global errors increase but the $J=1$ error is about three times larger than the $J=4$ error.

\section{Concluding remarks}

In this paper we have presented a new high-order sequence of NRBCs based on a reformulation of the Higdon NRBCs, for time-dependent wave problems. The new NRBCs involve no high derivatives, and are thus amenable for standard finite difference or finite element discretization. In this paper we considered the former; work on the latter is currently underway and will be reported elsewhere.

The new NRBCs involve auxiliary variables defined on the artificial boundary $\mathscr{B}$. On the other hand, they do not involve high derivatives. This allows the easy use of a NRBC of an arbitrarily high order. The scheme is coded once and for all for any order; the order of the scheme is simply an input parameter. Moreover, we have shown that the sequence of NRBCs is "converging" for a fixed $\mathscr{B}$, namely that by 

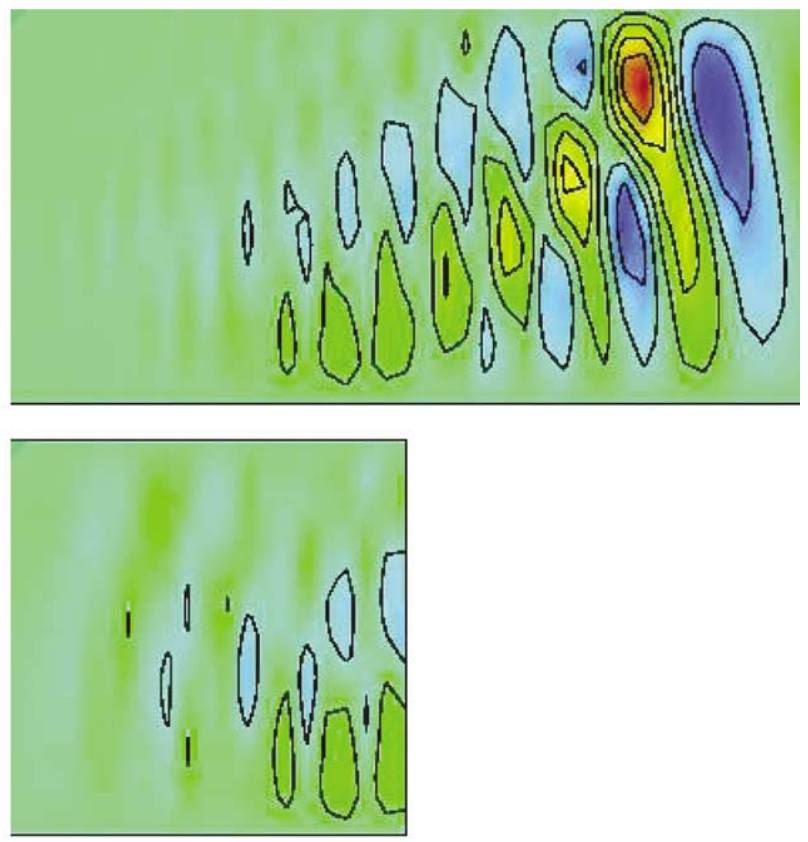

(e)

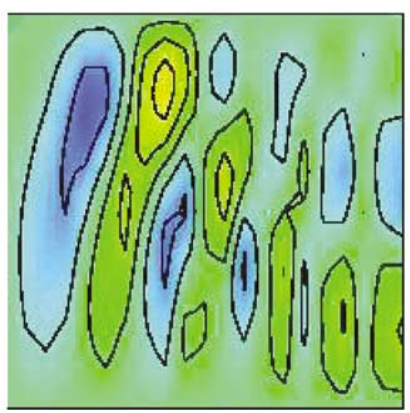

Fig. 7. (continued)

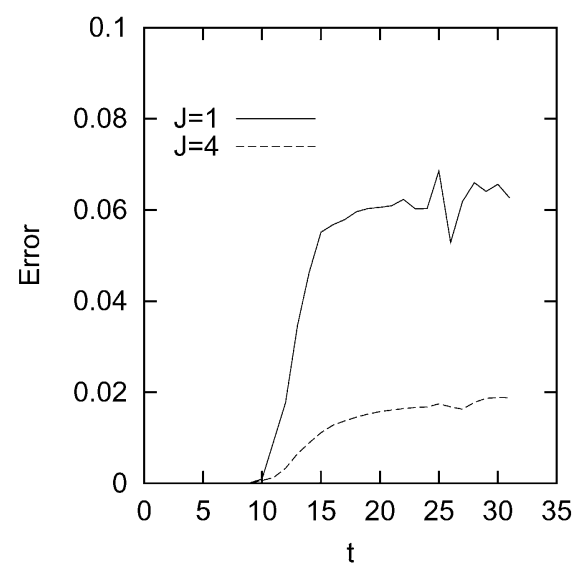

Fig. 8. The cosine-source problem: global error $E$ as a function of time, generated by the $J=4$ solution and by the $J=1$ solution. 
increasing the order one can achieve any desired level of accuracy. In addition, the computational effort associated with the new boundary treatment increases only linearly with the order, as opposed to the exponential growth in a previous scheme based on the original form of the Higdon NRBCs [46].

Due to the generality of the proposed NRBCs it is possible to use them for problems in dispersive and stratified media. We have demonstrated the good performance of the scheme for both non-dispersive and dispersive problems.

Related future work will include the adaptation of the proposed approach to more complicated configurations, such as exterior problems with a rectangular artificial boundary $\mathscr{B}$, and three-dimensional problems, all in the presence of wave dispersion. Exterior problems need to be investigated with special care, since in this case one has to deal with jump conditions and stability at the corner points, as mentioned in the Introduction. The new NRBCs will also be adapted to the case of curved artificial boundaries by using variable transformation; see, e.g. [60]. In addition, they will be applied to the shallow water equations (SWEs). These serve as an important testbed for more complicated dispersive models in meteorology [61].

\section{Acknowledgements}

This work was supported by the US National Research Council (NRC), the Office of Naval Research (ONR), and the Naval Postgraduate School (NPS).

\section{References}

[1] D. Givoli, Numerical Methods for Problems in Infinite Domains, Elsevier, Amsterdam, 1992.

[2] D. Givoli, Non-reflecting boundary conditions: a review, J. Comput. Phys. 94 (1991) 1-29.

[3] S.V. Tsynkov, Numerical solution of problems on unbounded domains, a review, Appl. Numer. Math. 27 (1998) $465-532$.

[4] D. Givoli, Exact representations on artificial interfaces and applications in mechanics, Appl. Mech. Rev. 52 (1999) $333-349$.

[5] T. Hagstrom, Radiation boundary conditions for the numerical simulation of waves, Acta Numer. 8 (1999) 47-106.

[6] B. Engquist, A. Majda, Radiation boundary conditions for acoustic and elastic calculations, Commun. Pure Appl. Math. 32 (1979) 313-357.

[7] A. Bayliss, E. Turkel, Radiation boundary conditions for wave-like equations, Commun. Pure Appl. Math. 33 (1980) $707-725$.

[8] J.B. Keller, D. Givoli, Exact non-reflecting boundary conditions, J. Comput. Phys. 82 (1989) 172-192.

[9] D. Givoli, J.B. Keller, Non-reflecting boundary conditions for elastic waves, Wave Motion 12 (1990) 261-279.

[10] V.S. Ryaben'kii, S.V. Tsynkov, Artificial boundary conditions for the numerical solution of external viscous flow problems, SIAM J. Numer. Anal. 32 (1995) 1355-1389.

[11] S.V. Tsynkov, E. Turkel, S. Abarbanel, External flow computations using global boundary conditions, AIAA J. 34 (1996) $700-706$.

[12] J.P. Bérenger, A perfectly matched layer for the absorption of electromagnetic waves, J. Comput. Phys. 114 (1994) 185-200.

[13] F. Collino, High order absorbing boundary conditions for wave propagation models. Straight line boundary and corner cases, in: R. Kleinman, et al. (Eds.), Proceedings of the 2nd International Conference on Mathematical and Numerical Aspects of Wave Propagation, SIAM, Delaware, 1993, pp. 161-171.

[14] M.J. Grote, J.B. Keller, Exact nonreflecting boundary conditions for the time dependent wave equation, SIAM J. Appl. Math. 55 (1995) 280-297.

[15] M.J. Grote, J.B. Keller, Nonreflecting boundary conditions for time dependent scattering, J. Comput. Phys. 127 (1996) 52-65.

[16] M.J. Grote, J.B. Keller, Nonreflecting boundary conditions for elastic waves, SIAM J. Appl. Math. 60 (2000) $803-819$.

[17] I.L. Sofronov, Conditions for complete transparency on the sphere for the three-dimensional wave equation, Russian Acad. Dci. Dokl. Math. 46 (1993) 397-401.

[18] T. Hagstrom, S.I. Hariharan, Progressive wave expansions and open boundary problems, in computational wave propagation, in: B. Engquist, G.A. Kriegsmann (Eds.), IMA Volumes in Mathematics and Its Applications, vol. 86, Springer, New York, 1997, pp. $23-43$.

[19] T. Hagstrom, S.I. Hariharan, A formulation of asymptotic and exact boundary conditions using local operators, Appl. Numer. Math. 27 (1998) 403-416. 
[20] M.N. Guddati, J.L. Tassoulas, Continued-fraction absorbing boundary conditions for the wave equation, J. Comput. Acoust. 8 (2000) 139-156.

[21] D. Givoli, High-order non-reflecting boundary conditions without high-order derivatives, J. Comput. Phys. 170 (2001) 849 870 .

[22] D. Givoli, I. Patlashenko, An optimal high-order non-reflecting finite element scheme for wave scattering problems, Int. J. Numer. Meth. Eng. 53 (2002) 2389-2411.

[23] E. Turkel, A. Yefet, Absorbing PML boundary layers for wave-like equations, Appl. Numer. Math. 27 (1998) $533-557$.

[24] F. Collino, P. Monk, The perfectly matched layer in curvilinear coordinates, SIAM J. Sci. Comp. 19 (1998) $2061-2090$.

[25] L. Ting, M.J. Miksis, Exact boundary conditions for scattering problems, J. Acoust. Soc. Am. 80 (1986) $1825-1827$.

[26] D. Givoli, D. Cohen, Non-reflecting boundary conditions based on Kirchhoff-type formulae, J. Comput. Phys. 117 (1995) 102113.

[27] A.J. Safjan, Highly accurate non-reflecting boundary conditions for finite element simulations of transient acoustics problems, Comput. Meth. Appl. Mech. Eng. 152 (1998) 175-193.

[28] D. Givoli, A spatially exact non-reflecting boundary condition for time dependent problems, Comput. Meth. Appl. Mech. Eng. 95 (1992) 97-113.

[29] I. Patlashenko, D. Givoli, P. Barbone, Time-stepping schemes for systems of volterra integra-differential equations, Comput. Meth. Appl. Mech. Eng. 190 (2001) 5691-5718.

[30] L.L. Thompson, P.M. Pinsky, Space-time finite element method for structural acoustics in infinite domains. Part 2: Exact timedependent non-reflecting boundary conditions, Comput. Meth. Appl. Mech. Eng. 132 (1996) 229-258.

[31] B. Alpert, L. Greengard, T. Hagstrom, Rapid evaluation of nonreflecting boundary kernels for time-domain wave propagation, SIAM J. Numer. Anal. 37 (2000) 1138-1164.

[32] B. Alpert, L. Greengard, T. Hagstrom, An integral evolution formula for the wave equation, J. Comput. Phys. 162 (2000) 536543.

[33] L.L. Thompson, R. Huan, Finite element formulation of exact nonreflecting boundary conditions for the time-dependent wave equation, Int. J. Numer. Meth. Eng. 45 (1999) 1607-1630.

[34] R. Huan, L.L. Thompson, Accurate radiation boundary conditions for the time-dependent wave equation on unbounded domains, Int. J. Numer. Meth. Eng. 47 (2000) 1569-1603.

[35] S. Krenk, Unified formulation of radiation conditions for the wave equation, Int. J. Numer. Meth. Eng. 53 (2002) $275-295$.

[36] J. Goodrich, T. Hagstrom, High order implementations of accurate boundary conditions, in: Proceedings of the 5th AIAA/CEAS Aeroacoustics Conference, Seattle, WA, AIAA, 1999.

[37] I.M. Navon, B. Neta, M.Y. Hussaini, A perfectly matched layer formulation for the nonlinear shallow water equations models: the split equation approach (to appear).

[38] D. Givoli, Recent Advances in the DtN finite element method for unbounded domains, Arch. Comput. Meth. Eng. 6 (1999) 71116.

[39] J. Pedlosky, Geophysical Fluid Dynamics, Springer, New York, 1987.

[40] R.L. Higdon, Radiation boundary conditions for dispersive waves, SIAM J. Numer. Anal. 31 (1994) 64-100.

[41] R.L. Higdon, Absorbing boundary conditions for difference approximations to the multi-dimensional wave equation, Math. Comput. 47 (1986) 437-459.

[42] R.L. Higdon, Numerical absorbing boundary conditions for the wave equation, Math. Comput. 49 (1987) 65-90.

[43] R.L. Higdon, Radiation boundary conditions for elastic wave propagation, SIAM J. Numer. Anal. 27 (1990) $831-870$.

[44] R.L. Higdon, Absorbing boundary conditions for elastic waves, Geophysics 56 (1991) 231-241.

[45] R.L. Higdon, Absorbing boundary conditions for acoustic and elastic waves in stratified media, J. Comput. Phys. 101 (1992) 386418.

[46] D. Givoli, B. Neta, High-order non-reflecting boundary conditions for dispersive waves, Wave Motion (in press).

[47] O. Vacus, Mathematical analysis of absorbing boundary conditions for the wave equation: the corner problem, preprint.

[48] R.A. Pearson, Consistent boundary conditions for the numerical models of systems that admit dispersive waves, J. Atmos. Sci. 31 (1974) 1418-1489.

[49] I. Orlanski, A simple boundary condition for unbounded hyperbolic flows, J. Comput. Phys. 21 (1976) $251-269$.

[50] W.H. Raymond, H.L. Kuo, A radiation boundary condition for multi-dimensional flows, Q. J. R. Meteorol. Soc. 110 (1984) 535551.

[51] M.J. Miller, A.J. Thorpe, Radiation conditions for the lateral boundaries of limited-area numerical models, Q. J. R. Meteorol. Soc. 107 (1981) 615-628.

[52] J.B. Klemp, D.K. Lilly, Numerical simulation of hydrostatic mountain waves, J. Atmos. Sci. 35 (1978) $78-107$.

[53] M. Wurtele, J. Paegle, A. Sielecki, The use of open boundary conditions with the Storm-Surge equations, Mon. Weather Rev. 99 (1971) $537-544$.

[54] R.M. Hodur, The naval research laboratory's coupled ocean/atmosphere mesoscale prediction system (COAMPS), Mon. Weather Rev. 125 (1997) 1414-1430. 
[55] X. Ren, K.H. Wang, K.R. Jin, Open boundary conditions for obliquely propagating nonlinear shallow-water waves in a wave channel, Comput. Fluids 26 (1997) 269-278.

[56] T.G. Jensen, Open boundary conditions in stratified ocean models, J. Marine Syst. 16 (1998) 297-322.

[57] F.S.B.F. Oliveira, Improvement on open boundaries on a time dependent numerical model of wave propagation in the nearshore region, Ocean Eng. 28 (2000) 95-115.

[58] J.C. Tannehill, D.A. Anderson, R.H. Pletcher, Computational Fluid Mechanics and Heat Transfer, second ed., Taylor \& Francis, Washington DC, 1997.

[59] B.P. Sommeijer, P.J. van der Houwen, B. Neta, Symmetric linear multistep methods for second order differential equations with periodic solutions, Appl. Numer. Math. 2 (1986) 69-77.

[60] D. Givoli, B. Neta, High-order Higdon non-reflecting boundary conditions for the shallow water equations, Report NPS-MA-02001, Naval Postgraduate School, Monterey, CA, 2002.

[61] D.R. Durran, Numerical Methods for Wave Equations in Geophysical Fluid Dynamics, Springer, New York, 1999. 